\title{
A Study of the Evolution of the Close Binaries Cyg X-3, IC 10 X-1, NGC 300 X-1, SS 433, and M33 X-7 Using the "Scenario Machine"
}

\author{
A. I. Bogomazov \\ M. V. Lomonosov Moscow State University, \\ P. K. Sternberg Astronomical Institute, \\ Moscow, Russia
}

\begin{abstract}
Evolutionary tracks for the X-ray binaries Cyg X-3, IC 10 X-1, NGC 300 X-1, SS 433, and M33 X-7 are computed using the Scenario Machine code. The compact objects in IC 10 X-1, NGC 300 X-1, and M33 X-7 are the most massive stellar mass black hole candidates. Cyg X-3, IC 10 X-1, and NGC 300 X-1 are the only currently known Wolf-Rayet stars with degenerate companions. SS 433 is the only known superaccretor in the Milky Way. Therefore, the stars studied provide excellent laboratories for testing scenarios for the evolution of binaries under extreme conditions. The classical evolutionary scenario is consistent with modern observational data. During the evolution of these binaries, hypernova explosions accompanied by the collapse of stellar cores with large angular momenta can occur, leading to long gammaray bursts. At the end of their evolution, Cyg X-3, IC 10 X-1, NGC 300 X-1, and SS 433 may form binary relativistic objects, which will subsequently merge due to the radiation of gravitational waves. The gravitational waves emitted during mergers of relativistic stars should be detectable by existing and future gravitational-wave antennas. In the course of its future evolution, M33 X-7 will pass through a Thorne-Zytkow stage. The formation of a Thorne-Zytkow object can also be accompanied by gravitational-wave radiation.
\end{abstract}

\section{Introduction}

The possibility of forming Wolf-Rayet stars in massive close binaries was considered in the classical paper [1]. Arguments suggesting the loss of mass and angular momentum in the course of the mass transfer preceding the formation of the Wolf-Rayet star were also provided in [1]. The evolution of such systems can include a stage with a close binary composed of a Wolf-Rayet star and a compact degenerate stellar remnant [2]. Currently, three X-ray binaries harboring Wolf-Rayet stars and stellar mass black hole candidates are known in the Milky Way and other nearby galaxies: Cyg X-3, IC 10 X-1, and NGC 300 $\mathrm{X}-1$. Certain aspects of the evolution of these three objects were studied in [3], together with the evolution of the close binary SS 433, which is a possible precursor to a Wolf-Rayet + compact remnant system, assuming that SS 433 will not undergo a common-envelope stage in its subsequent evolution (as it is currently in such a stage).

The aim of the present paper is to study the evolutionary paths of the massive close binaries Cyg X-3, IC 10 X-1, NGC 300 X-1, SS 433, and M33 X-7 using the "Scenario 
Machine"1. The choice of these systems is not coincidental. There are only three known black-hole candidates in binaries with Wolf-Rayet stars (Cyg X-3, IC 10 X-1, NGC 300 X-1), and their evolutionary path should go through several stages. SS 433 may be a precursor to a black hole + Wolf-Rayet binary, and is currently the only known superaccretor in the Milky Way. M33 X-7 is a close binary harboring a black-hole candidate (one of the most massive) and a slightly evolved non degenerate star. These systems can be considered to be "cornerstones" of the theory of the evolution of close binaries. Studies of their evolution enable verification of evolutionary scenarios for extreme conditions.

The evolution of close binaries such as those studied here can include the collapse of very massive stellar cores. Since they are components of binary systems, they can possess large angular momenta. Such events are usually associated with long gamma-ray burThe evolution of close binaries such as those studied here can include the collapse of very massive stellar cores. Since they are components of binary systems, they can possess large angular momenta. Such events are usually associated with long gamma-ray burst:2. Depending on the parameters of the evolutionary scenario, the evolution may end in the formation of a binary with two relativistic objects or of two single objects, resulting from the disruption of the system. If a close relativistic binary forms, its components will merge due to gravitational-wave radiation. Such a merger would produce an outburst of gravitational waves that should be detected by LIGO3. If one or both components of such a system is a neutron star, the merger will also produce a short gamma-ray burst.

Thus, studies of the evolution of Cyg X-3, IC 10 X-1, NGC 300 X-1, SS 433, and M33 X-7 are topical, since they enable the identification of the parameters of evolutionary scenarios and possible sources of gravitational waves and gamma-ray bursts (both long and short).

We will now briefly describe the systems studed. Cyg X-3 and SS 433 are Galactic objects, while IC $10 \mathrm{X}-1, \mathrm{NGC} 300 \mathrm{X}-1$, and M33 X-7 are located in the galaxies IC 10, NGC 300, and M33, respectively.

Cyg X-3 is a Wolf-Rayet star [9] paired with a compact object, which is believed to be a black hole (see, e.g., the review [10]). The orbital period of the system is $4.8 \mathrm{hr}$. In early studies, the compact object was usually taken to be a black hole with a mass from $7 M_{\odot}$ to However, later studies yielded a different result: the mass of the relativistic star does not exceed [12], admitting the presence of either a neutron star or a black hole in the system. According to the modern data on the Doppler shifts of X-ray lines, the mass of the compact object in Cyg X-3 does not exceed 3.6 $M_{\odot}$ [13. This still allows the possibility that this is a system with a black hole paired with a Wolf-Rayet star, but does not provide a final answer to its nature. An additional argument in favour of the hypothesis that there is a black hole in the system is that accreting neutron stars paired with Wolf-Rayet stars probably cannot exist, since their rotation would be strongly accelerated during the second episode of mass transfer, so that they would become either ejectors or propellers [14]. The X-ray luminosity of the Cyg X-3 system at 1-60 keV is $\sim 10^{38} \mathrm{erg} / \mathrm{s}$ and tahe bolometric luminosity of theWolfRayet star is $\approx 3 \cdot 10^{39} \mathrm{erg} / \mathrm{s}$.

\footnotetext{
${ }^{1} \mathrm{~A}$ code for population-synthesis studies of close binaries [4, 5].

${ }^{2}$ See, for instance, 6, 7], where the rate of Wolf-Rayet core collapses in extremely close binary systems is studied using the Scenario Machine.

${ }^{3}$ See, for instance, [8, where the rate of relativistic binary mergers is studied using the Scenario Machine.
} 
IC $10 \mathrm{X}-1$ consists of a Wolf-Rayet star and one of the most massive stellar black hole candidates [15]. The mass of the WolfRayet star is $32.7 \pm 2.6 M_{\odot}$, and the mass of the black hole estimated from the observed radial-velocity curve of the optical component is $23.1 \pm 2.1 M_{\odot}$ [16]. The orbital period of the system is $34.4 \mathrm{hr}$. IC $10 \mathrm{X}-1$ is a bright, variable X-ray source in the metal-poor galaxy IC 10, which is underoing a burst of star formation. The X-ray luminosity of the system is $\sim 10^{38} \mathrm{erg} / \mathrm{s} \mathrm{[17,} \mathrm{18].} \mathrm{The} \mathrm{most} \mathrm{probable}$ optical counterpart of the X-ray source is the bright Wolf-Rayet star [MAC92] 17A [19]. The nature of a synchrotron superbubble in IC 10 was studied in [20, 21], where it was shown that the most plausible mechanism for the formation of this bubble is a hypernova explosion. IC $10 \mathrm{X}-1$ is located inside this superbubble, and it is possible that formation of the two objects is related.

NGC 300 X-1 became the third known Wolf-Rayet binary with a degenerate companion [22]. The orbital period of the system is $32.8 \pm 0.4 \mathrm{hr}$. According to XMM Newton data, the mean observed luminosity of the system at $0.2-10 \mathrm{keV}$ is approximately $2 \cdot 10^{38}$ $\mathrm{erg} / \mathrm{s}$, reaching $\sim 10^{39} \mathrm{erg} / \mathrm{s}$ (taking into account absorption along the line of sight). According to $[23$ the radial-velocity semi-amplitude is $267 \pm 8 \mathrm{~km} / \mathrm{s}$, yielding a mass function of $2.6 \pm 0.3 M_{\odot}$, the orbital period is $32.3 \pm 0.2 \mathrm{hr}$. The spectroscopic mass of the optical component (WN Wolf-Rayet star) is $26_{-5}^{+7} M_{\odot}$, implying amass for the black hole of $20 \pm 4 M_{\odot}$ for the most likely inclination of the orbit, $60-75^{\circ}$. If only half of the optical continuum emission is provided by the Wolf-Rayet star, its mass is $15_{-2.5}^{+4} M_{\odot}$, and the mass of the black hole is $14.5_{-2.5}^{+3} M_{\odot}$.

SS 433 is the only known supercritical accretor in the Milky Way. Although this system has been known for a long time, and has been the subject of hundreds of papers, there is still ample scope for further studies. SS 433 is a close, eclipsing binary with an orbital period of about 13 days (see, e.g., the review [24]), in which the donor fills its Roche lobe and material overflows onto a relativistic component (black-hole candidate) on the thermal time scale, at an accretion rate of $\sim 10^{-4} M_{\odot} / \mathrm{yr}$. Estimates of the mass of the compact object range between 2 and $15 M_{\odot}$ [25]-[28], and do not enable unambiguous conclusions about the nature of the degenerate component.

M33 X-7 was discovered in the early 1980s [29]. Periodic variability of the X-ray source was discovered in [30, 31] and it was hypothesized that the source is one component of a binary. Later, an O6 III optical counterpart of M33 X-7 was found, which has minimum mass of $20 M_{\odot}$ and an orbital period of $3.45^{d}$ [32, 33]. Analysis of the observed radial-velocity curve of the X-ray binary using a Roche model and of the dependence of the component masses on the Roche-lobe filling factor was performed in [34]. For themost probablemass of the optical star, $70 M_{\odot}$, the mass of the compact object was found to be $15.55 \pm 3.20 M_{\odot}$, placing it among the most massive stellar black-hole candidates.

The evolution of IC 10 X-1 and M33 X-7 has already been studied using the Scenario Machine in [34, where the most characteristic evolutionary tracks were constructed. In our present study, we carry out additional studies of IC 10 X-1 and M33 X-7.

\section{Population synthesis}

Our studies of the origin and subsequent evolution of the close binary systems Cyg X-3, IC 10 X-1, NGC 300 X-1, SS 433, and M33 X-7 were carried out using the "Scenario 
Machine". This program is designed for studies of the evolution of close binaries through population-synthesis techniques, which can be applied to compute individual evolutionary tracks of close binaries and investigate the properties of groups of close binaries of various types. A detailed description of the "Scenario Machine" is provided in [4, 5], and the method used for the close-binary population synthesis is described, for instance, in the review paper [35]. In our present study, we took the problem to involve three free parameters: the rate of mass loss by the non-degenerate star, the fraction of the mass of the pre-supernova star that disappears beyond the event horizon during the formation of the black hole, and the efficiency of the common envelope stage. The values of other parameters were not varied, and were taken to be equal to their standard values during the computations. For each set of initial parameters, a population synthesis was performed for $10^{6}$ binary systems.

The stellar mass loss rate $\dot{M}$ is very important for two reasons: first, it significantly affects the semi-major axis of the binary and, second, it directly affects the mass of the star itself. We considered evolutionary scenario A from [5] only, in which mass loss by main sequence stars is described using the classical formula

$$
\dot{M}=\frac{\alpha L}{c V_{\infty}}
$$

where $L$ is the stellar luminosity, $V_{\infty}$ the stellarwind velocity at infinity, $c$ the speed of light, and $\alpha$ a free parameter. In scenario $\mathrm{A}$, the decrease in the stellar mass $\Delta M$ does not exceed $0.1\left(M-M_{\text {core }}\right)$ during any one evolutionary stage, where $M$ is mass of the star at the beginning of the stage and $M_{\text {core }}$ is the mass of the stellar core. We parametrized the mass-loss by Wolf-Rayet stars as $\Delta M_{W R}=\alpha M_{W R}$, where $M_{W R}$ is the maximum stellar mass in the Wolf-Rayet stage.

The mass of a black hole $M_{B H}$ formed by an exploding pre-supernova of mass $M_{p r e S N}$, was calculated as

$$
M_{B H}=k_{b h} M_{p r e S N},
$$

where the coefficient $k_{b h}$ is the fraction of the presupernova mass that disappears beyong the event horizon during the collapse, which was varied between 0.1 and 1.0.

During a common envelope stage, stars very efficiently transfer their angular momentum to the surrounding matter, and approach one another along a spiral trajectory. The efficiency of mass loss in the common envelope stage is described by the parameter $\alpha_{C E}=\Delta E_{b} / \Delta E_{\text {orb }}$, where $\Delta E_{b}=E_{\text {grav }}-E_{\text {thermal }}$ is the binding energy of the ejected envelope and $\Delta E_{\text {orb }}$ is the reduction in the orbital separation during the approach:

$$
\alpha_{C E}\left(\frac{G M_{a} M_{c}}{2 a_{f}}-\frac{G M_{a} M_{d}}{2 a_{i}}\right)=\frac{G M_{d}\left(M_{d}-M_{c}\right)}{R_{d}},
$$

where $M_{c}$ is the core mass of the mass losing star, which has initial mass $M_{d}$ and radius $R_{d}$ (this is a function of the initial semimajor axis $a_{i}$ and initial component-mass ratio $M_{a} / M_{d}$, where $M_{a}$ is the mass of the accretor).

In our computations, Cyg X-3, IC 10 X-1, and NGC 300 X-1 were taken to be black holes with Wolf-Rayet companions. Since the mass of the degenerate star is not known well, we considered two options: a low mass and a massive degenerate star. In the low 
mass version, the mass of the black hole was $\leq 10 M_{\odot}$, the mass of the Wolf-Rayet was $\leq 10 M_{\odot}$, the the orbital period of the system was $\leq 0.2$ day. In the high mass version, the mass of the black hole was $\geq 10 M_{\odot}$, the mass of the Wolf-Rayet star was $\geq 10 M_{\odot}$, the orbital period was $\leq 0.2$ day, and the X-ray luminosity was $\geq 10^{38} \mathrm{erg} / \mathrm{s}$. We adopted the following parameters for IC $10 \mathrm{X}-1$, in accordance with available observational data: mass of the black hole $23 \div 34 M_{\odot}$, mass of the Wolf-Rayet star $17 \div 35 M_{\odot}$, and the orbital period $\leq 1.5$ day. For NGC $300 \mathrm{X}-1$, we adopted the mass of the black hole to be $20 \div 25 M_{\odot}$, orbital period to be $\leq 1.5$ day, and $\mathrm{X}$ ray luminosity to be $\geq 10^{38} \mathrm{erg} / \mathrm{s}$; the mass of the Wolf-Rayet star was not constrained.

SS 433 was taken to be a black hole undergoing supercritical accretion paired with a non-degenerate star that overflows its Roche lobe. The mass of the optical star was taken to be $5 \div 15 M_{\odot}$.

M33 X-7 was taken to be a $14 \div 17 M_{\odot}$ black hole paired with a $65 \div 75 M_{\odot}$ star that is close to the end of its main-sequence evolution. The orbital period of the system was limited to 5 days.

\section{Results of the computations}

Results of the computations are presented in Figs. 1 1/5 and Tables 1 1 3, The main aim of our studies using the "Scenario Machine" is to determine the most probable range of parameters of evolutionary scenarios describing these systems. For this, we constructed a plot (Fig. 1) of the number of Cyg X-3 type and NGC 300 X-1 type systems (for the case of a massive black hole) in an $10^{11} M_{\odot}$ spiral galaxy with a Salpeter star formation rate as a function of the fraction of the mass of the presupernova that disappears beyond the event horizon during the formation of the black hole $k_{b h}$. The curves in Fig. 1 were computed for various efficiencies of the common-envelope stage $\alpha_{C E}$ and mass loss rates $\alpha$. Since each of the systems considered is the only object of its type in its parent galaxy, the main criterion for selecting a suitable set of evolutionary parameters was that the number of such systems be roughly equal to 1 in the model. If possible, the set of parameters satisfying all systems simultaneously was chosen. Figure 1 shows that this condition is satisfied for Cyg X-3 and NGC $300 \mathrm{X}-1$ for $\alpha_{C E}=0.5, a=0.3$, and $k_{b h}=0.5$. This set of parameters was used to compute the evolutionary tracks of the systems considered 4 .

The following notation is used for the evolutionary stages in Figs. 2 45 and Tables 1 1]3 (. [5]): I - main sequence star, II - supergiant, III, IIIe, IIIs - Roche lobe filling star, WR - Wolf-Rayet star, BB - Roche lobe filling Wolf-Rayet star, BH - black hole, $\mathrm{SBH}$ - superaccreting black hole, Psr - radio pulsar, CE - common envelope, and SN supernova explosion. The parameters presented in the figures and tables are: $M_{1}$ and $M_{2}$ - masses of primary and secondary components (in solar units), $a$ - semimajor axis of the orbit in $R_{\odot}, T$ - time elapsed since the formation of the system (in Myr), and $e-$ orbital eccentricity. All values of $M_{1}, M_{2}, a, T$, and $e$ are listed for the beginning of the corresponding stage; the time given for a supernovae is the time just before the explosion.

Figure 2 presents a characteristic evolutionary track for a binary resulting in the formation of a Cyg X-3 type system (massive black hole). At the beginning of the evolution,

\footnotetext{
${ }^{4}$ Similar plots were constructed for M33 X-7 type and IC 10 X-1 type in [34, Figs. 8 and 10.
} 
the mass of the primary (more massive) star is $M_{1}=60 \div 120 M_{\odot}$, the component-mass ratio is $q=\frac{M_{2}}{M_{1}}=0.3 \div 0.8$, and the initial semi-major axis of the orbit is $a=70 \div 140 R_{\odot}$. Note that the mass of a star can increase during mass transfer, so that the initial mass of a star may be lower than its mass in a subsequent evolutionary stage. After the depletion of hydrogen in the core, the primary star fills its Roche lobe. Matter begins to overflow onto the companion, as a rule, faster than the nuclear time scale (Stage III); the final phase of the overflow occurs on the nuclear time scale (Stage IIIe). The remnant of the primary after the loss of its envelope becomes a Wolf-Rayet star, which explodes and forms a black hole. Further, the secondary completes its main sequence evolution, becomes a supergiant, and, subsequently also fills its Roche lobe. In this configuration, the accretion rate becomes supercritical. A common envelope rapidly forms, the binary components become very close, and the envelope of the non-relativistic component is lost. A pair consisting of a black hole and a Wolf-Rayet star forms. The binary becomes so close that the Wolf-Rayet star may overflow its Roche lobe. During the collapse of a Wolf-Rayet star in such a binary, a long gamma-ray burst may occur. The final stage of the evolution of the system in this cast 5 is the merger of two black holes (the remnants of the components) due to gravitational-wave radiation, resulting in the formation of a single massive black hole. The merger of two black holes should produce an outburst of gravitational-wave radiation.

A characteristic evolutionary track leading to the formation of a Cyg X-3-type system with a lowmass black hole is presented in Fig. 3. At the beginning of the evolution, the mass of the primary star is $M_{1}=25 \div 37 M_{\odot}$, the component mass ratio is $q=$ $\frac{M_{2}}{M_{1}}=0.2 \div 0.9$, and the initial semimajor axis of the orbit is between $a=30$ and $130 R_{\odot}$. After the depletion of hydrogen in the core, the primary becomes a supergiant and subsequently fills its Roche lobe. The stellar material overflows from the lobe on a time scale that is shorter than the nuclear time scale (Stage III). After the loss of the envelope, the primary becomes a Wolf-Rayet star, which leaves a black hole remnant after its explosion as a supernova. Next, the secondary finishes its main sequence evolution, becomes a supergiant, and later overflows its Roche lobe. The accretion rate becomes supercritical. A common envelope rapidly forms, in which the components become very close. The envelope of the non-relativistic component is lost. A binary "black hole + Wolf-Rayet" system forms. A long gamma ray burst can also occur during the collapse of the Wolf-Rayet star in such a binary. Since the component masses are lower in this scenario than in the scenario described above, as a rule, the remnant of the secondary is a neutron star rather than of a black hole.

Depending on an additional parameter - the kick obtained by the neutron star when it is born, such a system can produce either a single black hole and a single neutron star (which will manifest itself as a radio pulsar at the beginning of its lifetime), or a binary system containing a black hole and neutron star. In the latter case, it is possible that the pair is so close that the black hole and neutron star will merge due to the radiation of gravitational wave, producing a short gamma-ray burst and an outburst of gravitational waves. However, investigation of the influence of the possible kick accompanying the birth of the neutron star is beyond the scope of this paper.

Figure 4 presents a characteristic evolutionary track of a binary leading to the forma-

\footnotetext{
${ }^{5}$ We assumed that the black holes did not receive a significant kick during their formation.
} 
tion of an NGC $300 \mathrm{X}-1$ type system. At the beginning of the evolution, the mass of the primary is $M_{1}=90 \div 120 M_{\odot}$, the component mass ratio is $q=\frac{M_{2}}{M_{1}}=0.35 \div 0.75$, and the initial semi-major axis of the orbit is $a=70 \div 160 R_{\odot}$. After hydrogen depletion in the core, the primary star fills its Roche lobe. Mass transfer onto the companion begins, which, as a rule, proceeds on a time scale shorter than the nuclear time scale (Stage III). The loss of the primary's envelope results in the formation of a Wolf-Rayet star, which forms a black hole when it explodes as a supernova. Further, after finishing its main sequence evolution, the secondary overflows its Roche lobe. A common envelope rapidly forms, in which the components become very close. The envelope of the non-relativistic component is lost. A black hole and Wolf-Rayet star in a pair is formed. The collapse of the Wolf-Rayet star may be accompanied by a long gamma ray burst. The result of the evolution of this binary is the merger of two black holes remnants due to gravitational wave radiation, with the formation of a single massive black hole. The merger of two black holes produces an outburst of gravitational wave radiation.

Figure 5presents an example of one track from a family of possible evolutionary tracks of binaries that result in the formation of IC $10 \mathrm{X}$-1-type systems 6 . At the beginning of the evolution, the mass of the primary is $M_{1}=75 \div 120 M_{\odot}$, the component mass ratio is $q=\frac{M_{2}}{M_{1}}=0.15 \div 0.6$, and the initial semimajor axis of the orbit is $a=70 \div 280 R_{\odot}$. After depletion of hydrogen in the core, the primary overflows its Roche lobe, and forms a common envelope around the two non-degenerate stars. In this stage, the components become very close, and the envelope of the primary is partially lost and partially accreted by the secondary. After the loss of the primary's envelope, a WolfRayet star forms. The binary becomes so close that the secondary overflows its Roche lobe before the depletion of hydrogen in its core. After the explosion of the Wolf-Rayet primary and the collapse of its core into a black hole, a binary system containing a black hole and a star filling its Roche lobe forms. Accretion proceeds on the nuclear time scale (Stage IIIe). After the loss of its envelope, the secondary becomes a Wolf-Rayet star, which subsequently explodes and forms a neutron star. The system is already so close at the time of the first supernova explosion that the formation of the black hole may be accompanied by a gamma-ray burst7.

After the loss of the secodary's envelope, it likewise becomes a Wolf-Rayet star. The explosion of the secondary produces a neutron star, which is manifest as a radio pulsar at the beginning its evolution. If the kick obtained by the newborn neutron star is not large, the resulting binary harbors a black hole and a radio pulsar, which later fades. If the newborn neutron star does not receive a significant kick, the system is wide enough to avoid a merger of the components over the Hubble time (this option is presented in Fig. 5). A kick could also to disruption of the system or a reduction in the semi-major axis of the orbit, with the latter resulting in the merger of the components due to gravitationalwave radiation over a reasonable time. In the latter case, a short gamma ray burst and an outburst of gravitational wave radiation could occur.

Three possible and approximately equally probable evolutionary tracks for the SS

\footnotetext{
${ }^{6}$ The track shown Fig. 5 supplements the track presented in Fig. 11 in 34, where calculations were carried out with a slightly different set of parameters, in particular, a lower mass-loss rate.

${ }^{7} \mathrm{~A}$ gamma ray burst can probably occur during the collapse of the core of the Wolf-Rayet star if the orbital period of the binary is $\lesssim 0.5$ day [7].
} 
433 system are presented in Tables 1, 3. For a very wide range of parameters in the evolutionary scenarios, the number of SS 433-type systems in the Galaxy significantly exceeds unity, and is equal to 10-20. Thus, other systems similar to SS 433 should exist in the Milky Way ${ }^{8}$. The initial parameters of the precursors of SS 433 type systems is poorly constrained. The initial masses of the stars must be such that the primary could form a black hole after its explosion as a supernova (i.e., at some stage of its evolution before the supernova, its mass must exceed $25 M_{\odot}$ ), and the initial semimajor axis of the orbit must enable the overflow of the secondary's Roche lobe. The initial component mass ratio can vary over a very wide range: 0.1-0.9.

The evolution of the system most similar to SS 433 is presented in the Table 1. The primary of this very close binary fills its Roche lobe before the depletion of hydrogen in its core. The time scale for mass transfer onto the secondary is initially shorter than the nuclear time scale (Stage III), but becomes comparable to the nuclear time scale at the end of the stage (Stage IIIe). After the loss of its envelope, the primary becomes a WolfRayet star. Further, the secondary fills its Roche lobe before the completion of hydrogen burning in its core. The time scale for the mass transfer onto the primary is initially shorter than the nuclear time scale, but then becomes similar to the nuclear time scale. After the explosion of the primary as a supernova, a black hole undergoing supercritical accretion is formed. We identify this stage with an SS 433 type star. The age of the nebula surrounding SS 433, which was apparently formed in a supernova explosion, W50, does not exceed $10^{5} \mathrm{yr}$ (see, for instance, 38] and references therein). This makes the track presented in the Table 1, most plausible as a description of the evolutionary path followed by SS 433. After the loss of its envelope in the process of supercritical accretion, the optical star becomes a Wolf-Rayet star. After the explosion of the secondary, the system becomes a binary black hole. The tracks presented in Tables 2 and 3 exhibit twomain differences compared to the track in the Table 1. First, after the first supernova explosion in the system, the secondary fills its Roche lobe several hundred thousand years after the explosion as it leaves the main sequence, which does not agree with the age of W50. Second, a common envelope stage begins after the SS 433 stage, supplementing the possible paths for the subsequent evolution of the SS 433 type systems considered in [3].

Our study of the evolution of the M33 X-7 system assuming various evolutionary parameters did not reveal additional evolutionary scenarios compared to those illustrated in Fig. 9 in [34]. This is true because the parameters of M33 X-7 are known much more accurately than those of the other systems considered here. Therefore, the ranges of possible component masses and orbital semimajor axes used in our calculations were the narrowest for this system. Thus, M33 X-7 stillmost tightly constrains the evolutionary scenarios for the systems considered systems. In the evolutionary models included in the Scenario Machine, the evolutionary track for M33 X-7 has two significant differences from the other tracks: first, it is not possible to obtain an M33 X-7-type system with appreciably higher mass loss rates by the non-degenerate stars $\left(\alpha=0.3, k_{W R}=0.3\right)$, and, second, the most probable fraction of pre-supernova mass that disappears beyond

\footnotetext{
${ }^{8}$ Uncertainty in the masses of the SS 433 components means that we cannot definitively rule out a neutron star as the compact component of the system. The observational manifestations of a magnetized neutron star in a system with supercritical accretion are similar to the properties of SS 433 [36], and the number of the binaries classified as "super accreting neutron star + Roche lobe filling non-degenerate star" in the Galaxy should also reach 20 [37.
} 
the event horizon, $k_{b h}$, is less than 0.5 , and approximately equal to 0.3 .

\section{Conclusions}

We have investigated possible evolutionary tracks for the X-ray binaries Cyg X-3, IC 10 X-1, NGC 300 X-1, SS 433, and M33 X-7 using the "Scenario Machine". Each of these systems can be considered a potential source of gravitational-wave radiation, and can potentially produce one to three gamma ray bursts in the course of its evolution? 9 . During the evolution of SS 433, after the stage corresponding to the current state of this system, a binary harboring a Wolf-Rayet star and a black hole should form, with properties close to those of Cyg X-3, NGC 300 X-1, and IC 10 X-1. In Figs. 2,5, the evolutionary stages preceeding the formation of these systems are also similar to SS 433, but have higher masses. Unlike the results of [3], SS 433 and the precursors of Cyg X-3, NGC 300 X-1, and IC $10 \mathrm{X}-1$ can have a stage with common envelope after the stage corresponding to the current state of SS 433. Some of the evolutionary tracks presented here include a "radio pulsar + black hole" stage. The discovery of such a system would provide firm confirmation of the existence of stellar mass black holes [41].

It follows from Figs. 155 and Tables 1-3 that the origins of Cyg X-3, IC 10 X-1, NGC $300 \mathrm{X}-1$, and SS 433 can be described using classical evolutionary scenarios. The main difference between classical evolutionary scenarios and the scenarios presented, for example, in [42]-44] 10, is a much lower rate of mass loss by massive stars via their stellar winds. It is shown in [39] that the winds of massive stars may be clumpy. This means that observational estimates of the wind mass loss rates should be reduced by a factor of three to five. In addition, the most massive of the systems considered (M33 X-7, IC 10 $\mathrm{X}-1$, and NGC $300 \mathrm{X}-1$ ) reside in galaxies with lower metallicities than the Milky Way (see, e.g., 45] for M33, [46] for IC 10, and [47] for NGC 300). Decreasing the metallicity also means decreasing the mass loss rate. Thus, classical evolutionary scenarios with low mass loss rates, including via stellar winds of massive stars, have a fairly strong physical basis.

The evolution of Cyg X-3 was studied by Zdziarski et al. [48] who concluded that the degenerate component of Cyg X-3 is either a low mass black hole (due to the accretioninduced collapse of a neutron star or the collapse of a stellar core during its supernova explosion) or a neutron star. In the theory underlying the Scenario Machine, a neutron star cannot be manifest as an accretor if it is in close pair with a Wolf-Rayet star [14]. In addition, as a rule, an accreting neutron star may accumulate enough mass to collapse into a black hole only if its companion is a long lived, low mass star [49]. The precursors of Wolf-Rayet stars cannot play this role.

\footnotetext{
${ }^{9} \mathrm{Up}$ to two long bursts and a short burst in the case of IC $10 \mathrm{X}-1$. According to [7] a gamma ray burst can occur during the first supernova explosion in a system with the parameters given in Fig. 5 , whereas this is not possible according to [40] since the Wolf-Rayet star must be in a binary system with a degenerate companion. In this case, a gamma ray burst will occur only after the second supernova explosion. However, the existence of a bubble around IC $10 \mathrm{X}-1$ supports the possibility of a gamma-ray burst already after the first supernova explosion [20, 21.

${ }^{10}$ The evolution of M33 X-7 and IC 10 X-1 was studied in [42] of M33 X-7 in [43] and of IC $10 \mathrm{X}-1$ and NGC $300 \mathrm{X}-1$ in [44].
} 
We have considered two sets of the main parameters for Cyg X-3-type systems: corresponding to high-mass and low-mass black holes. Observational data supporting the low mass option are provided in [48]. However, the results of calculations carried out in our current study contradict the conclusions of [48]. In particular, the low-mass option implies that the X-ray luminosity of the system generated by the physical mechanisms assumed in the "Scenario Machine" should be a factor of ten lower than the real luminosity of Cyg X-3. At the same time, the number of low-mass Cyg X-3-type systems in the Galaxy should be $\sim 10$. If our code is incorrectly estimating the X-ray luminosity, this would suggest that nine out of ten very bright X-ray sources in the Galaxy had not been discovered, which seems implausible, in view of the data provided by the "Integral" space observatory and many other observations. Thus, our calculations predict the existence of up to $\sim 10$ systems consisting of a Wolf-Rayet star and a fairly low mass black hole with X-ray luminosities $\lesssim 10^{37} \mathrm{erg} / \mathrm{s}$. Apparently, Cyg X-3 must be fairly massive, suggesting that the high mass option for the evolutionary path of the system is more realistic than the low mass one.

This study was supported by the Russian Foundation for Basic Research (project 12-02-31301 mol-a).

\section{References}

1. A. V. Tutukov and L. R. Yungelson, Nauchn. Inform. Astron. Sovet AN SSSR 27, 58 (1973).

2. A. V. Tutukov and L. R. Yungelson, Nauchn. Inform. Astron. Sovet AN SSSR 27, 70 (1973).

3. A. V. Tutukov, A. V. Fedorova, and A. M. Cherepashchuk, Astron. Rep. 57, 667 (2013).

4. V. M. Lipunov, K. A. Postnov, and M. E. Prokhorov, Astrophys. and Space Phys. Revs 9, 1 (1996).

5. V. M. Lipunov, K. A. Postnov, M. E. Prokhorov, and A. I. Bogomazov, Astron. Rep. 53, 915 (2009).

6. A. I. Bogomazov, V. M. Lipunov, and A. V. Tutukov, Astron. Rep. 51, 308 (2007).

7. G. V. Lipunova, E. S. Gorbovskoy, A. I. Bogomazov, V. M. Lipunov, Mon. Not. Roy. Astron. Soc. 397, 1695 (2009).

8. V. M. Lipunov, K. A. Postnov, M. E. Prokhorov, Mon. Not. Roy. Astron. Soc. 288, 245 (1997).

9. M. H. van Kerkwijk, T. R. Geballe, D. L. King et al., Astron. Astrophys. 314, 521 (1996).

10. A. M. Cherepashchuk, Phys. Usp. 46, 335 (2003). 
11. W. Schmutz, T. R. Geballe, H. Schild, Astron. Astrophys. 311, L25 (1996).

12. M. M. Hanson, M. D. Still, R. P. Fender, Astrophys. J. 541, 308 (2000).

13. M. J. Stark, M. Saia, Astrophys. J. 587, L101 (2003).

14. V.M. Lipunov, Sov. Astron. Lett. 8, 194 (1982).

15. A. H. Prestwich, R. Kilgard, P. A. Crowther, et al., Astrophys. J. 669, L21-L24 (2007).

16. J. M. Silverman, A. V. Filippenko, Astrophys. J. 678, L17 (2008).

17. W. N. Brandt, M. J. Ward, A. C. Fabian, P. W. Hodge, Mon. Not. Roy. Astron. Soc. 291, 709 (1997).

18. F. E. Bauer, W. N. Brandt, Astrophys. J. 601, L67 (2004).

19. P. A. Crowther, L. Drissen, J. B. Abbott, et al., Astron. Astrophys. 404, 483 (2003).

20. T. A. Lozinskaya, A. V. Moiseev, Mon. Not. Roy. Astron. Soc. 381, L26 (2007).

21. T. A. Lozinskaya, A. V. Moiseev, N. Yu. Podorvanyuk, and A. N. Burenkov, Astron. Lett. 34, 217 (2008).

22. S. Carpano, A. Pollock, A. Prestwich, et al., Astron. Astrophys. 466, p. L17 (2007).

23. P. Crowther, R. Barnard, S. Carpano, et al., Mon. Not. Roy. Astron. Soc. 403, p. L41 (2010).

24. S. Fabrika, Astrophys. Space Phys. Rev. 12, 1 (2004).

25. S. N. Fabrika, L. V. Bychkova, Astron. Astrophys. 240, L5 (1990).

26. T. Hillwig, D. Gies, W. Huand, et al., Astrophys. J. 615, 422 (2004).

27. K. Blundell, M. Bowler, L. Schmidtobreick, Astrophys. J. 678, L47 (2008).

28. K. Kubota, Y. Ueda, S. Fabrika, et al., Astrophys. J. 709, 1374 (2010).

29. K. S. Long, S. Dodorico, P. A. Charles, M. A. Dopita, Astrophys. J. (Letters) 246, L61 (1981).

30. G. Peres, F. Reale, A. Collura, and G. Fabbiano, Mem. Soc. Astron. Ital. 60, 221 (1989).

31. G. Peres, F. Reale, A. Collura, and G. Fabbiano, Astrophys. J. 336, 140 (1989).

32. 7. W. Pietsch, B. J. Mochejska, Z. Misanovic, et al., Astron. and Astrophys. 413, 879 (2004).

33. W. Pietsch, F. Haberl,M. Sasaki, et al., Astrophys. J. 646, 420 (2006). 
34. M. K. Abubekerov, E. A. Antokhina, A. I. Bogomazov, and A. M. Cherepashchuk, Actron. Zh. 86, 260 (2009).

35. S. B. Popov and M. E. Prokhorov, Phys. Usp. 50, 1123 (2007).

36. V. M. Lipunov and N. I. Shakura, Sov. Astron. 26, 386 (1982).

37. V. M. Lipunov, Astrophysics of Neutron Stars (Nauka, Moscow, 1987; Springer, Heidelberg, 1992).

38. P. Goodall, F. Alouani-Bibi, K. Blundell, Mon. Not. Roy. Astron. Soc. 414, 2838 (2011).

39. A. M. Cherepashchuk, Kh. Khaliullin, and J. A. Eaton, Astrophys. J. 281, 774 (1984).

40. E. P. J. van den Heuvel, S.-C. Yoon, Astrophysics and Space Science 311, 177-183 (2007).

41. V. M. Lipunov, A. I. Bogomazov, M. K. Abubekerov, Mon. Not. Roy. Astron. Soc. 359, 1517 (2005).

42. S. E. de Mink, M. Cantiello, N. Langer, et al., Astron. Astrophys. 497, 243 (2009).

43. F. Valsecchi, E. Glebbeek, W. M. Farr, et al., Nature 468, i. 7320, 77 (2010).

44. T. Bulik, K. Belczynski, A. Prestwich, Astrophys. J. 730, 140 (2011).

45. J. A. Orosz, J. E. McClintock, R. Narayan, et al., Nature 449, 872 (2007).

46. P. Massey, K. A. G. Olsen, P. W. Hodge, et al., Astron. J. 133, 2393 (2007).

47. F. Bresolin, W. Gieren, R.-P. Kudritzki, et al., Astrophys. J. 700, 309 (2009).

48. A. Zdziarski, J. Mikoajewska, K. Belczynski, Mon. Not. Roy. Astron. Soc. 429, L104 (2013).

49. A. I. Bogomazov, M. K. Abubekerov, V. M. Lipunov, and A. M. Cherepashchuk, Astron. Rep. 49, 295 (2005). 
Table 1: Evolutionary track for SS 433 most closely describing the evolution of the system. For notation, see Section 3 .

\begin{tabular}{|c|c|c|c|c|c|}
\hline & $M_{1}$ & $M_{2}$ & $a$ & $T$ & $e$ \\
\hline I+I & 66.71 & 38.94 & 31 & 0 & 0 \\
III+I & 64.43 & 35.65 & 33 & 2.7 & 0 \\
IIIe+I & 50.04 & 50.04 & 27 & 2.7 & 0 \\
WR+I & 35.80 & 60.34 & 33 & 3.3 & 0 \\
WR+III & 35.66 & 60.34 & 33 & 3.3 & 0 \\
WR+IIIe & 44.33 & 44.33 & 30 & 3.3 & 0 \\
SN & 45.03 & 39.70 & 32 & 3.6 & 0 \\
SH+III & 22.51 & 39.70 & 50 & 3.6 & 0.36 \\
BH+WR & 22.51 & 31.11 & 33 & 3.6 & 0.05 \\
SN & 22.51 & 21.78 & 40 & 3.9 & 0.05 \\
BH+BH & 22.51 & 10.89 & 62 & 3.9 & 0.37 \\
\hline
\end{tabular}

Table 2: Additional evolutionary track for an SS 433 type system. For notation, see Section 3 .

\begin{tabular}{|c|c|c|c|c|c|}
\hline & $M_{1}$ & $M_{2}$ & $a$ & $T$ & $e$ \\
\hline I+I & 38.71 & 27.87 & 89 & 0 & 0 \\
II+I & 33.63 & 25.86 & 99 & 4.2 & 0 \\
III+I & 33.50 & 25.86 & 99 & 4.2 & 0 \\
IIIE+I & 27.06 & 27.06 & 100 & 4.2 & 0 \\
WR+I & 16.71 & 29.66 & 150 & 4.2 & 0 \\
BB+I & 11.70 & 29.44 & 170 & 4.6 & 0 \\
SN & 10.60 & 29.55 & 200 & 4.6 & 0 \\
BH+I & 5.30 & 29.55 & 230 & 4.6 & 0.15 \\
BH+II & 5.30 & 29.09 & 240 & 5.4 & 0.15 \\
SH+IIIs & 5.30 & 18.33 & 340 & 5.9 & 0 \\
CE & 5.30 & 16.46 & 220 & 5.9 & 0 \\
BH+WR & 5.30 & 11.51 & 180 & 5.9 & 0 \\
SN & 5.30 & 8.06 & 220 & 6.4 & 0 \\
BH+BH & 5.30 & 4.03 & 400 & 6.4 & 0.43 \\
\hline
\end{tabular}


Table 3: Additional evolutionary track for an SS 433 type system. For notation, see Section 3,

\begin{tabular}{|c|c|c|c|c|c|}
\hline & $M_{1}$ & $M_{2}$ & $a$ & $T$ & $e$ \\
\hline I+I & 60.38 & 42.86 & 280 & 0.0 & 0.00 \\
II+I & 58.10 & 37.31 & 300 & 3.4 & 0.00 \\
III+I & 52.72 & 36.94 & 320 & 3.8 & 0.00 \\
WR+I & 31.13 & 46.77 & 380 & 3.8 & 0.00 \\
WR+II & 24.90 & 46.40 & 410 & 3.9 & 0.00 \\
SN & 22.42 & 40.58 & 470 & 4.0 & 0.00 \\
BH+II & 11.21 & 40.58 & 600 & 4.0 & 0.22 \\
SH+IIIs & 11.21 & 26.22 & 800 & 4.3 & 0.15 \\
CE & 11.21 & 24.30 & 700 & 4.3 & 0.14 \\
BH+WR & 11.21 & 21.78 & 190 & 4.3 & 0.00 \\
SN & 11.21 & 15.24 & 230 & 4.7 & 0.00 \\
BH+BH & 11.21 & 7.62 & 390 & 4.7 & 0.41 \\
\hline
\end{tabular}



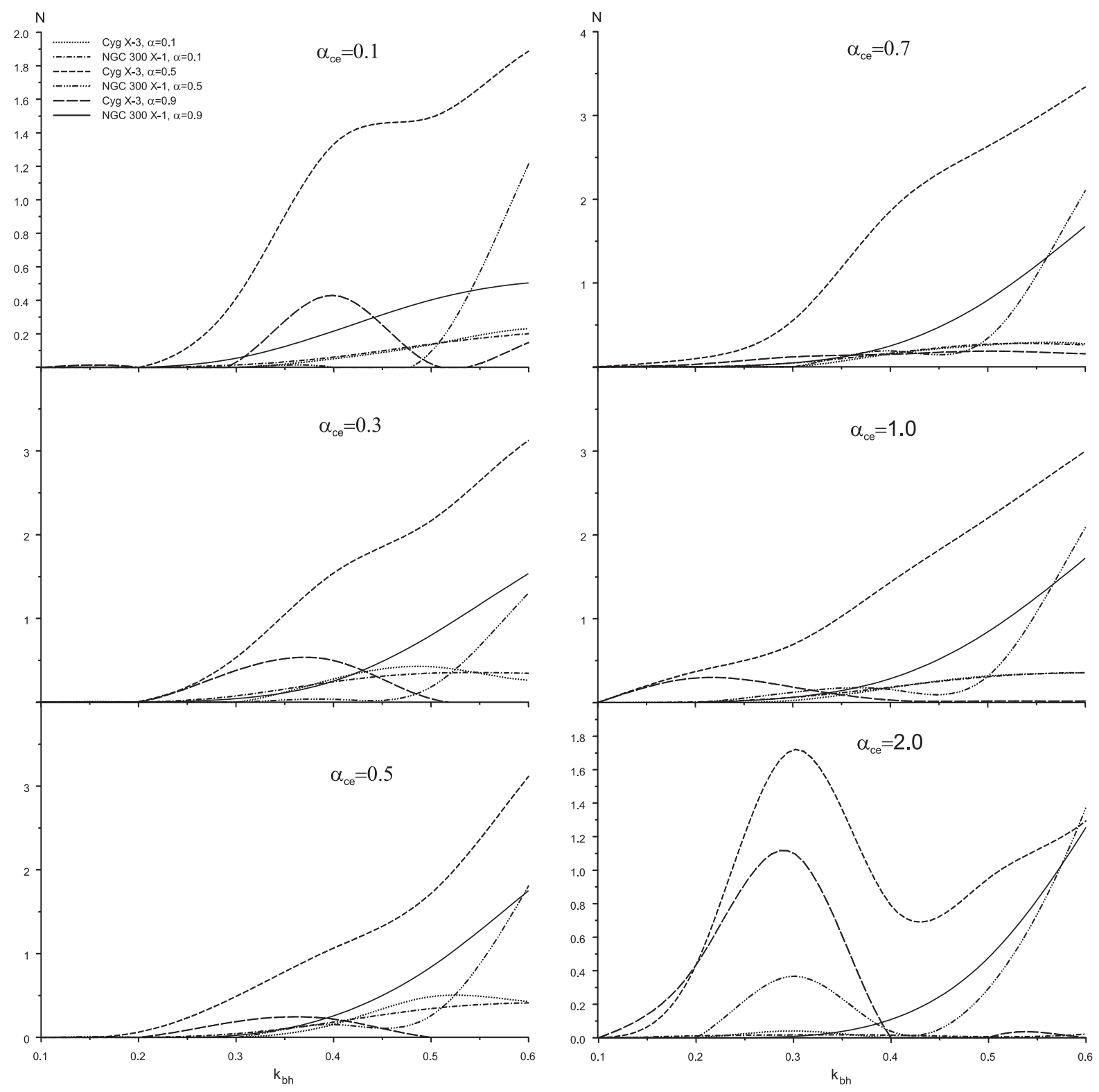

Figure 1: Number of Cyg X-3 and NGC 300 X-1-type systems in a $10^{11} \mathrm{M}_{\odot}$ spiral galaxy with a Salpeter star formation rate as a function of the fraction of the pre-supernova mass disappearing beyond the event horizon during the formation of the black hole, $k_{b h}$. The curves were computed for different efficiencies of the common envelope stage $\alpha_{C E}$ and mass loss rates $\alpha$. 


\begin{tabular}{|c|c|c|c|c|c|}
\hline система & $M_{1}$ & $M_{2}$ & a & $\mathrm{T}$ & e \\
\hline $1+1$ & 69.23 & 40.73 & 110 & 0 & 0 \\
\hline$|I|+\mid$ & 66.75 & 37.03 & 120 & 2.6 & 0 \\
\hline IIle+I & 50.96 & 50.96 & 100 & 2.6 & 0 \\
\hline WR+IIle & 37.71 & 53.51 & 120 & 3.3 & 0 \\
\hline SN & 26.40 & 53.39 & 140 & 3.5 & 0 \\
\hline $\mathrm{BH}+\mathrm{I}$ & 13.2 & 53.39 & 180 & 3.5 & 0.2 \\
\hline $\mathrm{BH}+\mathrm{II}$ & 13.2 & 53.13 & 180 & 4.1 & 0.2 \\
\hline $\mathrm{SBH}+\mathrm{IIIS}$ & 13.2 & 52.97 & 170 & 4.1 & 0.1 \\
\hline SBH+III, CE & 13.2 & 47.89 & 100 & 4.1 & 0.1 \\
\hline $\mathrm{BH}+\mathrm{WR}$ & 13.2 & 26.29 & 3.6 & 4.1 & 0 \\
\hline $\mathrm{BH}+\mathrm{BB}$ & 13.2 & 18.40 & 4.5 & 4.4 & 0 \\
\hline SN & 13.2 & 16.83 & 4.5 & 4.4 & 0 \\
\hline $\mathrm{BH}+\mathrm{BH}$ & 13.2 & 8.41 & 7.4 & 4.4 & 0.4 \\
\hline $\mathrm{BH}$ & & & & 93 & \\
\hline
\end{tabular}

Figure 2: Evolutionary scenario for the formation of a Cyg X-3-type system (massive black hole). The notation is described in Section 3 , 


\begin{tabular}{|c|c|c|c|c|c|}
\hline система & $\mathrm{M}_{1}$ & $\mathrm{M}_{2}$ & a & $\mathrm{T}$ & e \\
\hline $1+1$ & 32.32 & 19.73 & 79 & 0 & 0 \\
\hline $\mid I+I$ & 28.86 & 18.99 & 86 & 4.7 & 0 \\
\hline $\mid I I+I$ & 21.44 & 18.94 & 100 & 5.2 & 0 \\
\hline$W R+I I I$ & 12.98 & 20.39 & 140 & 5.2 & 0 \\
\hline SN & 9.08 & 20.31 & 160 & 5.6 & 0 \\
\hline $\mathrm{BH}+\mathrm{I}$ & 4.54 & 20.31 & 200 & 5.6 & 0.2 \\
\hline $\mathrm{BH}+\mathrm{II}$ & 4.54 & 19.92 & 200 & 7.9 & 0.2 \\
\hline $\mathrm{SBH}+\mathrm{IIIS}$ & 4.54 & 17.35 & 220 & 8.6 & 0.1 \\
\hline $\mathrm{SBH}+\mathrm{III}, \mathrm{CE}$ & 4.54 & 15.64 & 140 & 8.6 & 0.1 \\
\hline $\mathrm{BH}+\mathrm{WR}$ & 4.54 & 6.81 & 3.1 & 8.6 & 0 \\
\hline SN & 4.54 & 4.77 & 3.7 & 9.3 & 0 \\
\hline $\mathrm{BH}+\mathrm{Psr}$ & 4.54 & 1.34 & 9.1 & 9.3 & 0.6 \\
\hline
\end{tabular}

Figure 3: Evolutionary scenario for the formation of a Cyg X-3-type system (low-mass black hole). The notation is described in Section 3 . 


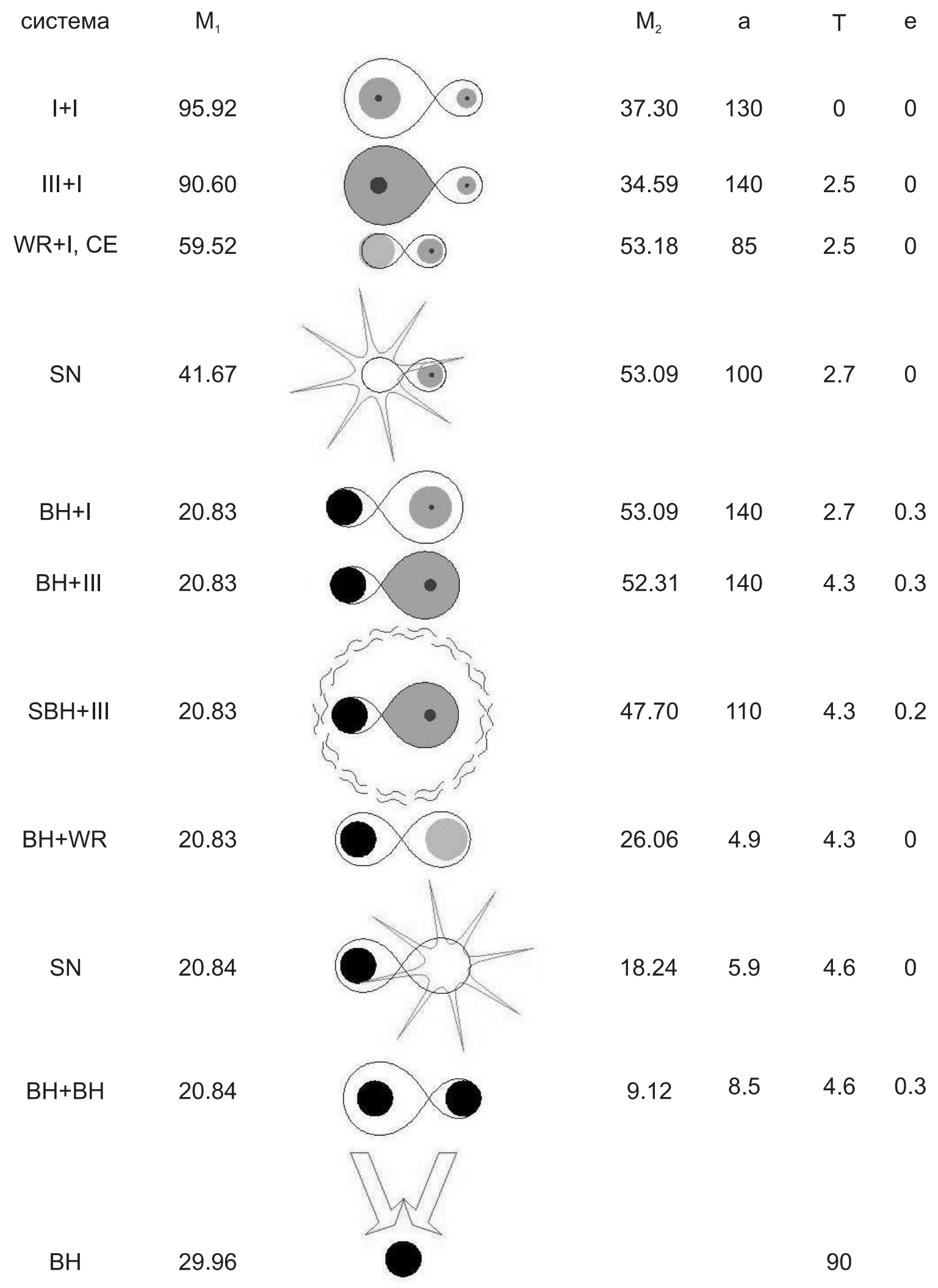

Figure 4: Evolutionary track for NGC $300 \mathrm{X}-1$. The notation is described in Section 3 , 


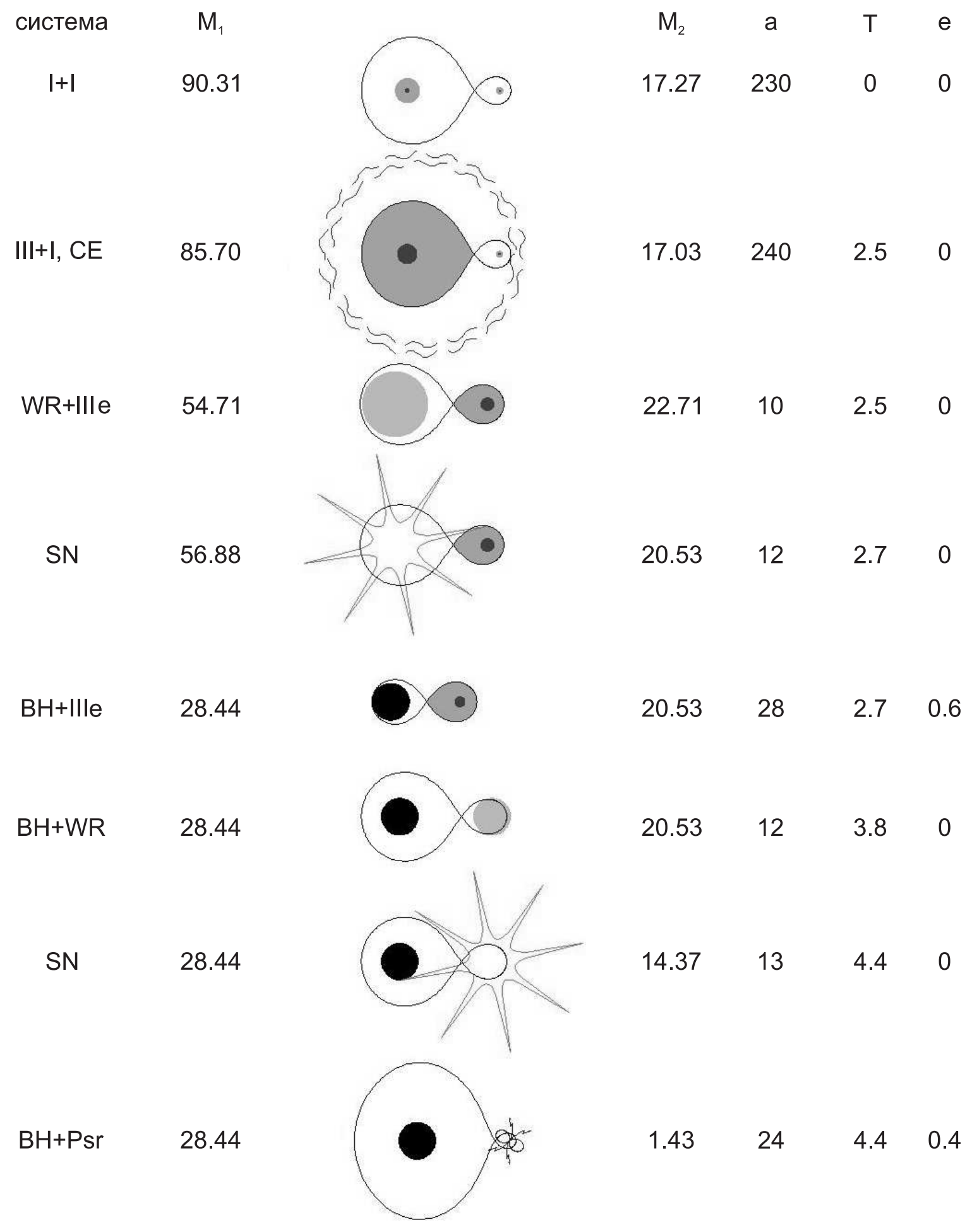

Figure 5: Evolutionary track for IC $10 \mathrm{X}-1$. The notation is described in Section 3 . 